\title{
ARTICLE
}

Received 26 Nov 2012 | Accepted 21 Mar 2013 | Published 7 May 2013

DOI: $10.1038 /$ ncomms 2797

OPEN

\section{A universal description of ultraslow glass dynamics}

\author{
Julio Cesar Martinez-Garcia', Sylwester J. Rzoska²,3, Aleksandra Drozd-Rzoska ${ }^{4} \&$ Jorge Martinez-Garcia ${ }^{5}$
}

The dynamics of glass is of importance in materials science but its nature has not yet been fully understood. Here we report that a verification of the temperature dependencies of the primary relaxation time or viscosity in the ultraslowing/ultraviscous domain of glass-forming systems can be carried out via the analysis of the inverse of the Dyre-Olsen temperature index. The subsequent analysis of experimental data indicates the possibility of the selfconsistent description of glass-forming low-molecular-weight liquids, polymers, liquid crystals, orientationally disordered crystals and Ising spin-glass-like systems, as well as the prevalence of equations associated with the 'finite temperature divergence'. All these lead to a new formula for the configurational entropy in glass-forming systems. Furthermore, a link to the dominated local symmetry for a given glass former is identified here. Results obtained show a new relationship between the glass transition and critical phenomena.

\footnotetext{
${ }^{1}$ Department of Chemistry and Biochemistry, University of Berne, Freiestrasse 3, Berne CH-3012, Switzerland. ${ }^{2}$ Institute of Physics, University of Silesia, ul. Uniwersytecka 4, Katowice 40-007, Poland. ${ }^{3}$ Institute of High Pressure Physics, Polish Academy of Sciences, ul. Sokołowska 27/39, Warsaw 01-143, Poland. ${ }^{4}$ Foundation C2B, ul. Wolności 3/5, Chorzów 41-500, Poland. ${ }^{5}$ Lucerne University of Applied Sciences, Technikumstrasse 21, Horw CH-6048, Switzerland. Correspondence and requests for materials should be addressed to J.C.M.-G. (email: jmartinezgarcia@gmail.com).
} 


\section{T} he glass transition constitutes one of the outstanding problems of condensed matter physics ${ }^{1}$, with fundamental importance for many areas ranging from geophysics to modern food science and material engineering ${ }^{2-5}$. Over the last decades, an important progress in this field has been achieved, primarily because of the progress in the state-of-the-art experimental, theoretical and numerical insights. Hence, a conceptual breakthrough seems to emerge ${ }^{6-18}$.

Recently, however, serious doubts regarding the most fundamental experimental artifact, namely the parameterization of viscosity $\eta(T)$ or primary relaxation time $\tau(T)$ on approaching the glass temperature $\left(T_{\mathrm{g}}\right)$, appeared ${ }^{15,19-21}$. The importance of this issue is related to the fact that it is often associated with a hypothetical link between the glass transition and critical phenomena physics, that is, the thrilling possibility of a new great unification within condensed matter physics ${ }^{14,18}$. Moreover, relations portraying the evolution of $\eta(T)$ or $\tau(T)$ are often considered as a checkpoint for theoretical models ${ }^{3,13}$. Decades of studies have led to the prevailing conviction that the ultimate parameterization is possible via the Vogel-Fulcher-Tammann (VFT) equation $3,13,22-24$ :

$$
\tau(T)=\tau_{0} \exp \left(\frac{D_{T} T_{0}}{T-T_{0}}\right) \text { and } \eta(T)=\eta_{0} \exp \left(\frac{D_{T} T_{0}}{T-T_{0}}\right)
$$

where $D_{T}$ denotes the fragility strength coefficient and $T_{0}$ is the VFT singular temperature $\left(T_{0} \ll T_{\mathrm{g}}\right.$ and $\left.T>T_{\mathrm{g}}\right)$.

However, by following the compelling analysis of 43 sets of $\tau(T)$ data for low-molecular-weight ultraviscous liquids, Hecksher et al. ${ }^{15}$ recently concluded: 'There is no compelling evidence for the VFT prediction that the relaxation time diverges at a finite temperature [...] with a dynamic divergence of the VFT form lack a direct experimental basis.... we suggest that in the search for the correct theory for ultraviscous liquid dynamics, theories not predicting a dynamic divergence of the VFT form should be focused on'. McKenna ${ }^{25}$ extended this outlook to the case of polymer glass formers. These findings were strengthened by the derivation by Mauro et al. ${ }^{19}$ of the equation introduced empirically by Waterton ${ }^{26}$ :

$$
\tau(T)=\tau_{0} \exp \left[\frac{K}{T} \exp \left(\frac{C}{T}\right)\right]
$$

The subsequent analysis of experimental data confirmed the advantage of the Waterton-Mauro (WM, called also MYEGA) equation without a 'finite temperature divergence' over the VFT one $e^{11,19-21,27}$. Thus, the question regarding $\tau(T)$ or $\eta(T)$ portrayal seemed to be clarified. However, the problem becomes more complex if glass-forming liquid crystals (LCs) and orientationally disordered crystals (ODICs; plastic crystals) are also considered. For these materials, the prevalence of the 'critical-like' equation with the clear 'finite temperature divergence' was clearly shown 28,29 :

$$
\tau(T)=\tau_{\mathrm{C}}\left(\frac{T-T_{\mathrm{C}}}{T_{\mathrm{C}}}\right)^{-\phi}
$$

where $T>T_{\mathrm{g}}$ and $T_{\mathrm{C}}<T_{\mathrm{g}}$ : most often $T_{\mathrm{g}}-T_{0} \approx 30-40 \mathrm{~K}$ and $T_{\mathrm{g}}-T_{\mathrm{C}} \approx 10-15 \mathrm{~K}$.

Surprisingly, the latter description seems to be applicable also for selected low-molecular-weight liquids and polymers ${ }^{28,29}$. Worth mentioning is that such dynamics is also commonly accepted for spin-glass-like (SGL) systems ${ }^{5,28}$. Remarkable is furthermore the approximate coincidence of power exponents in the mentioned systems, namely $\phi \approx 9$ for $\mathrm{LC}^{28,29}, \phi=9-15$ in $\mathrm{ODIC}^{28,29}$ and $\phi=9-12$ in $\mathrm{SGL}^{3,13,28}$.

In this respect, it is important to point out the link between anomalous dynamics in supercooled glass-forming systems and the cooperatively rearranged regions, introduced by the Adam and Gibbs (AG) model $3,13,30$, leading to the following output relation $^{30}$ :

$$
\tau(T)=\tau_{0} \exp \left(\frac{A \Delta \mu}{T S_{\mathrm{C}}}\right)
$$

where $\Delta \mu$ defines that the free-energy barrier between cooperatively rearranged region and $S_{\mathrm{C}}$ is the configurational entropy related to the difference between the entropy of the metastable disordered supercooled system and the stable crystal state.

Angell et al. ${ }^{31-33}$ noted that the AG equation can be converted into the VFT one by assuming:

$$
S_{\mathrm{C}}(T)=S_{0}\left(\frac{T-T_{0}}{T}\right)
$$

for $T_{0}=T_{\mathrm{K}}$, where the latter denotes the Kauzmann temperature ${ }^{34}$.

Although equation (5) became popular in the semi-phenomenological analysis of supercooled glass formers 3,13 , some puzzling issues remain. Mauro ${ }^{11,19}$ has shown that the 'divergence-less' equation (2) can also be derived from the AG dependence by omitting equation (5) for the configurational entropy. Moreover, the analysis of experimental data indicated that the coincidence between $T_{0}$ and $T_{\mathrm{K}}$ may be limited ${ }^{35}$. Eckmann and Procaccia ${ }^{36}$ showed that for a soft potential based model, the configurational entropy can stay positive for $T>0$, concluding that the Kauzmann temperature may not exist.

Particularly noteworthy is that the parameterization of $\tau(T)$ or $\eta(T)$ via 'finite temperature divergence' relations was used as an important argument to support the hypothesis, which correlates the glass transition with a hidden phase transition below $T_{\mathrm{g}}$. For instance, Colby ${ }^{16}$ suggested that equation (3), with exponent $\phi=9$, may be 'universal' for glass-forming low-molecular-weight liquids and polymers, although for some of them, a multiplicative factor $\exp (E / k T)$ should be included. The 'critical-like' divergence was associated with a hypothetical phase transition at $T_{\mathrm{C}}<T_{\mathrm{g}}$, linked to the correlation length $\xi(T)=\xi_{0}\left(T-T_{\mathrm{C}}\right)^{-v}$ with $v=3 / 2$ (refs 16,17). However, a subsequent thorough analysis of $\tau(T)$ experimental data did not confirm the suggested superiorfitting features ${ }^{28,29,37}$. Notwithstanding, there are several lowmolecular-weight and polymeric glass formers for which the behaviour suggested by Colby holds surprisingly well ${ }^{28,29}$.

It is worth mentioning that in 2010, Tanaka et al. ${ }^{18}$ carried out an in-depth simulation analysis of six glass-forming systems and stated: 'Our results suggest a far more direct link than thought before between glass transition and critical phenomena. Indeed, the glass transition may be a new type of critical phenomenon where a structural order parameter is directly linked to slowness'. Tanaka et al. ${ }^{18}$ linked the 'pretransitional' behaviour of the relaxation time to the Ising-like critical power-law divergence of the correlation length towards the ideal glass transition with the exponent $v \approx 2 / d$, where $d$ defines the spatial dimensionality. This led to the VFT-type equation $\tau(T)=\tau_{\infty} \exp \left[D_{T} T_{0} /\right.$ $\left.\left(T-T_{0}\right)\right]=\tau_{\infty} \exp \left[D_{T}\left(\xi(T) / \xi_{0}\right)^{d / 2}\right.$. Tanaka et al. ${ }^{18}$, further suggested that glass transition may be encountered in the 'Ising universality class for systems with a non-conserved order parameter'.

At this point, some basic concepts of critical phenomena physics are worth recalling ${ }^{38,39}$. The pretransitional behaviour for different physical magnitudes $(X(T))$ is described by the relation $X(T) \propto\left|T-T_{\mathrm{C}}\right|^{\lambda}$, where the values of critical exponents $(\lambda)$ are universal and depend solely on the dimensionality of the order parameter, the space dimensionality and the range of interactions. The symmetry is playing an essential role, whose changes are quantified by the order parameter. Microscopically, different 
systems can be ascribed in universality classes. It is also worth to bear in mind that the physics of critical phenomena is considered one of the greatest 'unification' successes in condensed matter physics of the 20th century $2,38,39$. To the best of the authors' knowledge, no clear manifestation of the above fundamental features for the glass-transition phenomenon has been reported so far. This paper aims to fill this gap and resolve the Gordian knot of the $\tau(T)$ or $\eta(T)$ evolution on approaching the glass transition.

\section{Results}

Fragility and the Dyre-Olsen temperature index. The SuperArrhenius (SA) evolution of relaxation time or viscosity in the ultraslowing/ultraviscous domain can be generally expressed as $\tau(T)=\tau_{\infty} \exp \left[E_{\mathrm{a}}^{\prime}(T) / R T\right]$, where $E_{\mathrm{a}}(T)=E_{\mathrm{a}}^{\prime}(T) / R$ stands for the normalized apparent activation energy (AAE) and $R$ denotes the gas constant ${ }^{3,13}$. The departure from the simple Arrhenius behaviour with $E_{\mathrm{a}}=$ const is characterized by the fragility coefficient $(m)$, introduced by Angell et al. ${ }^{40,41}$ as the metric at the normalized plots $\log _{10} \tau$ or $\log _{10} \eta$ versus $T_{\mathrm{g}} / T$ :

$$
\begin{aligned}
& m=m_{\mathrm{P}}\left(T=T_{\mathrm{g}}\right)=\left[\frac{\mathrm{d} \log _{10} \tau(T)}{\mathrm{d}\left(T_{\mathrm{g}} / T\right)}\right]_{T \rightarrow T_{\mathrm{g}}}, \\
& m=m_{\mathrm{P}}\left(T=T_{\mathrm{g}}\right)=\left[\frac{\mathrm{d} \log _{10} \eta(T)}{\mathrm{d}\left(T_{\mathrm{g}} / T\right)}\right]_{T \rightarrow T_{\mathrm{g}}}
\end{aligned}
$$

Although the fragility coefficient $m$ provides a metric for glassforming liquids within a wide diversity of materials, it is not the only parameter of this type ${ }^{3,13}$. A decade ago, Dyre-Olsen (DO) ${ }^{42}$ noted the formal importance of the Grüneisen parameter $(\gamma)^{43}$ :

$$
\gamma_{i}=-\frac{V}{\omega_{i}} \frac{\partial \omega_{i}}{\partial V}=-\frac{\partial \ln \omega_{i}}{\partial \ln V}
$$

It indicates that if the restoring force acting on an atom is nonlinear in displacement, the individual phonon frequency $\omega_{i}$ changes with the volume of crystal $V$, and parameter $\gamma$ reaches a transition from zero (linear displacement) to $\gamma>0$ (ref. 43). In the $\mathrm{SA}$ domain, the AAE increases with a decreasing temperature, allowing a dramatic increase from constant values to $\partial E_{\mathrm{a}}(T) / \partial T>0$, hence giving rise to a non-linear behaviour in the $\log _{10} \tau$ versus $1 / T$ representation. This formal similarity allowed to propose the metric of the SA behaviour by the 'Grüneisen-style' activation energy temperature index ${ }^{42}$ :

$$
I_{D O}(T)=-\frac{d \ln E_{a}(T)}{d \ln T}
$$

The DO temperature index can serve as the alternative metric of fragility, which can be also presented as $m=m\left(T=T_{\mathrm{g}}\right)=\left(H_{\mathrm{a}}^{\prime}\left(T_{\mathrm{g}}\right) / T_{\mathrm{g}}\right) \log _{10} e, \quad$ where $H_{\mathrm{a}}^{\prime}(T)=H_{\mathrm{a}} / R$ and $H_{\mathrm{a}}(T)=\mathrm{d} \ln \tau / \mathrm{d}(1 / T)$ denote the apparent activation enthalpy ${ }^{44}$.

Assuming the hypothetical 'universal' value for the prefactor $\tau_{0}=10^{-14} \mathrm{~s} \quad($ refs $3,13,15)$, one obtains: $m=\log _{10}\left[\tau\left(T_{\mathrm{g}}\right) / \tau_{0}\right]\left(I_{\mathrm{DO}}\left(T_{\mathrm{g}}\right)+1\right)=16\left[I_{\mathrm{DO}}\left(T_{\mathrm{g}}\right)+1\right], \quad$ for $\tau\left(T_{\mathrm{g}}\right)=10^{2} \mathrm{~s}$ and $\tau_{0}=10^{-14} \mathrm{~s}$. Subsequently, taking into account the recent suggestion that $16<m<175$ (ref. 45), one can estimate: $0<I_{\mathrm{DO}}\left(T_{\mathrm{g}}\right)<10$. It is noteworthy that in 1997 , Simon et al. ${ }^{46}$ indicated the upper fragility limit $m=214$, yielding $I_{\text {DO }}\left(T_{\mathrm{g}}\right)=12.375$. Very recently, McKenna ${ }^{47}$ indicated that if $T_{0}$ is an ideal glass transition, the value of $m$ evaluated at $T=T_{0}=T_{\mathrm{g}}$ would tend towards infinity for all materials. The same can be expected for $I_{\mathrm{DO}}\left(T_{\mathrm{g}}\right)$.

Heksher et al. ${ }^{15}$ showed that the analysis of $I_{\mathrm{DO}}(T)$ may represent a decisive tool for testing the validity of the given equation for fitting $\tau(T)$ experimental data in low-molecular- weight glass-forming ultraviscous liquids. To quantify how exactly the VFT and the popular Bässler-Avramov ${ }^{48,49}$ (BA) $\tau(T)=\tau_{0} \exp \left(A / T^{\mathrm{D}}\right)$ equations describe the data, they compared experimental and 'theoretical' values of $I_{\mathrm{DO}}(T)$, namely ${ }^{15}$ :

$$
I_{\mathrm{DO}}^{\mathrm{VFT}}(T)=\frac{T_{0}}{T-T_{0}} \quad I_{\mathrm{DO}}^{\mathrm{BA}}(T)=D-1=\mathrm{const}
$$

Derivations of $I_{\mathrm{DO}}(T)$ for equations (2) and (3) are presented in the Methods section. In the ultraslowing domain, near $T_{\mathrm{g}}$, they are as follows:

$$
I_{\mathrm{DO}}^{\mathrm{WM}}(T)=\frac{C}{T} \quad I_{\mathrm{DO}}^{\text {Crit. }}(T) \approx \frac{\phi}{T-T_{\mathrm{C}}}
$$

A glimpse on equations (9) and (10) shows that reciprocals of DO indexes yield a linear dependence $I_{\mathrm{DO}}^{-1}(T)=b+a T$, namely:

$$
\frac{1}{I_{\mathrm{DO}}(T)}=\left\{\begin{array}{l}
\left(\frac{1}{T_{0}}\right) T-1 \rightarrow(\mathrm{VFT}) \\
\frac{1}{D-1} \rightarrow(\mathrm{BA}) \\
\left(\frac{1}{\mathrm{C}}\right) T \rightarrow(\mathrm{WM}) \\
\left(\frac{1}{\phi}\right) T-\frac{T_{\mathrm{c}}}{\phi} \rightarrow \text { (Crit.) }
\end{array}\right.
$$

The discussed evolutions of DO indexes are shown graphically in Fig. 1. Two characteristic features are worth mentioning, namely: $I_{\mathrm{DO}}^{-1}(T=0)=b$ and $I_{\mathrm{DO}}^{-1}\left(T_{\mathrm{C}}, T_{0}\right)=0$. It is worth stressing that while the physics associated with the fragility index $m$ has been very broadly discussed $3,13,15,20,31,40-45,50,51$, the meaning and properties of the DO index is poorly explored so far ${ }^{15,42}$.

For the estimation of $I_{\mathrm{DO}}(T)$, the evolution of $E_{\mathrm{a}}(T)$ has to be determined. This, however, constitutes a notable issue. Hecksher et al. ${ }^{15}$ calculated $E_{\mathrm{a}}(T)$ from the $\mathrm{SA}$ relation, assuming $\tau_{0}=10^{-14} \mathrm{~s}$ as an 'universal averaged' value of the prefactor. However, in the systems analysed in this paper $\tau_{0}=10^{-11} s \div 10^{-16} s$ (refs $28,29,44$ ). In the Methods section, we present a novel way of determining $E_{\mathrm{a}}(T)$, avoiding the biasing influence of poorly estimated values of the prefactor $\tau_{0}$ for the given system.

Experimental data analysis. This paper focuses on the ultraslow/ ultraviscous domain as in Hecksher et al. ${ }^{15}$, for $\tau>\tau\left(T_{\mathrm{B}}\right) \approx$ $10^{-7} \mathrm{~s}$ or $\eta>\eta\left(T_{\mathrm{B}}\right)>10^{3}$ Poise, which usually occurs for $T_{\mathrm{B}}-T_{\mathrm{g}}<80 \mathrm{~K}$ (refs $50-53$ ). By heating above $T_{\mathrm{B}}$, the so-called dynamic cross-over occurs. The discussion on recent advances regarding this phenomenon can be found in Martinez-Garcia et al. ${ }^{52}$ The analysis of experimental data presented below does not only cover the most often discussed supercooled lowmolecular-weight liquids (L) and multimeric and polymeric (P) glass formers as in Hecksher et al. ${ }^{15}$, but also LCs, plastic crystals (ODICs) and SGL systems. The analysis is based on 16 selected sets of $\tau(T)$ experimental data representing these types of glassforming systems. However, to clarify the discussion, the key results are presented for a smaller number of characteristic systems. They are L including diethyl phthalate (DEP), glycerol, sorbitol, ethanol ${ }^{28,54}, \mathrm{P}$ including EPON 828 and polystyrene $\left(M_{\mathrm{W}}=700\right)$ (refs 28,55), ODIC (plastic crystal) including $\mathrm{C7}-\mathrm{OH}$, $\mathrm{C} 8-\mathrm{OH}$ (ref. 53), CNc6 (ref. 57), $\mathrm{CNadm}^{58,59}$ and $\mathrm{NPaNPG}_{0.3}$ (ref. 56), and SGL including ferrofluid liquid, with $5 \%$ solution of single-domain particles of the amorphous alloy $\mathrm{Fe}_{1-} \mathrm{C}_{x}$ $(x=0.2-0.3)$ (ref. 57). Details regarding all tested systems are collected in the Supplementary Table S1. The presented analysis is focused on the primary relaxation time $\tau(T)$, because the modern broad-band dielectric spectroscopy can yield sets of high precision and consistent experimental data ${ }^{3,13}$. For the presentation of experimental data, we apply the linearized derivative-based analysis introduced in Drozd-Rzoska and 

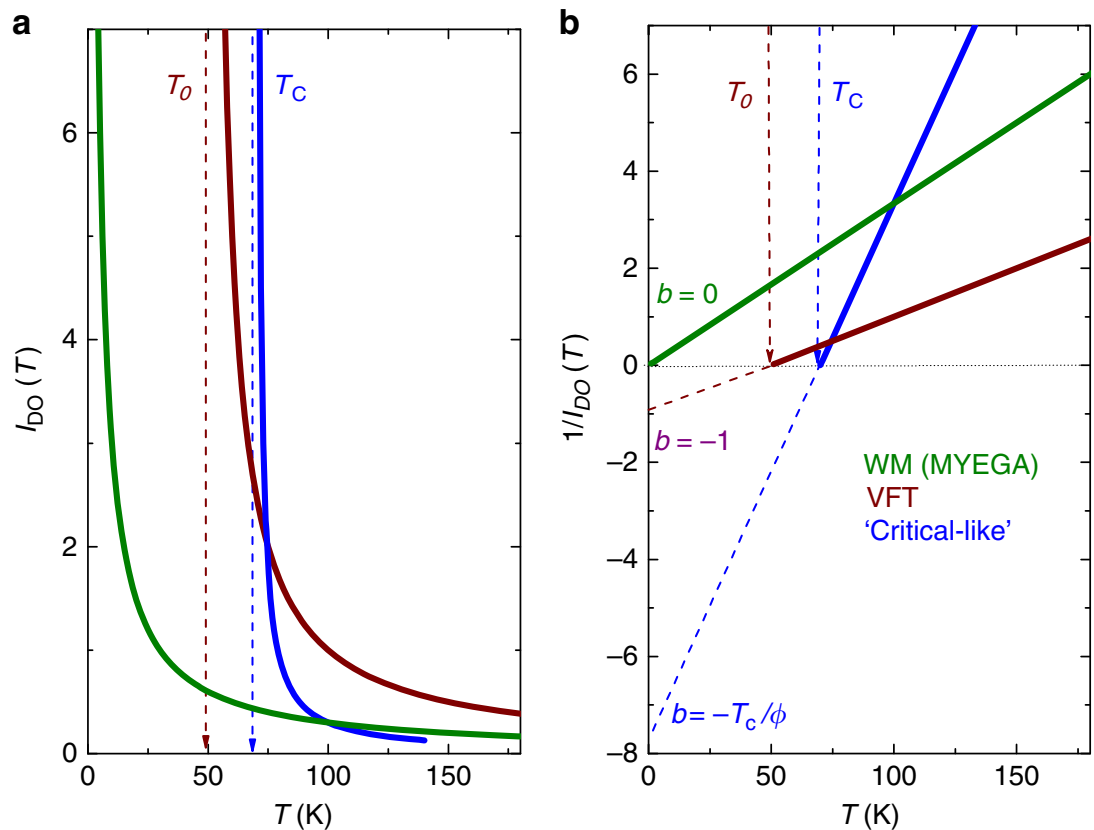

Figure 1 | Evolution of the DO temperature index for different equations portraying $\boldsymbol{\tau}(\boldsymbol{T})$ behaviour. (a,b) Evolutions of DO index for the WM (MYEGA) (green line), VFT (wine line) and 'critical-like' dependences (blue line). The omitted BA equation yields a horizontal line.

Rzoska $^{44}$. The VFT equation allows the following transformation of $\tau(T)$ data:

$$
\begin{aligned}
{\left[\frac{\mathrm{d} \ln \tau}{\mathrm{d}(1 / T)}\right]^{-1 / 2} } & =\frac{H_{\mathrm{a}}(1 / T)}{R}=\left[H_{\mathrm{a}}^{\prime}(1 / T)\right]^{-1 / 2} \\
& =\left[\left(D_{T} T_{o}\right)^{-1 / 2}\right]-\frac{\left[T_{0}\left(D_{T} T_{0}\right)^{-1 / 2}\right]}{T}=B-\frac{A}{T}
\end{aligned}
$$

For the critical-like equation (3):

$$
\frac{T^{2}}{H_{\mathrm{a}}^{\prime}(T)}=\frac{T-T_{\mathrm{C}}}{\phi}=A T-B
$$

where $H_{\mathrm{a}}^{\prime}(T)$ stands for the normalized apparent enthalpy.

The linear behaviour of equations (12) and (13) show the valid temperature domain of the VFT and 'critical-like' equations. The subsequent linear regression fit can yield optimal values of relevant parameters ${ }^{44}$. Results of such analysis for selected systems are shown in Fig. 2a-e. It is visible that a clear 'critical-like' parameterization is preferred for all LC rod-like compounds, as well as for sorbitol, ethanol and polystyrene (Fig. 2b). This behaviour can be linked to the uniaxial structure of molecules, which may lead to the local orientational symmetry (OS) arrangements ${ }^{28}$. The 'critical-like' behaviour also dominates the dynamics of plastic crystals (ODICs) $^{28,29}$, where a positional symmetry (PS) is a fundamental feature (Fig. 2a). Regarding EPON 828, glycerol and DEP, the critical-like parameterization definitively fails (Fig. 2c). Because these compounds cannot be linked neither to OS nor to PS cases, we assigned them as no-symmetry (NS) compounds.

For 'NS' glass formers, the VFT equation fits the experimental data fairly well as shown in Fig. 2e. It is noteworthy that for ethanol and sorbitol, both equations (1) and (3) seem to offer the acceptable parameterization (Fig. 2b,d).

For a further insight into the optimal $\tau(T)$ parameterization, an analysis exploring $I_{\mathrm{DO}}^{-1}(T)$ behaviour has been carried out (Fig. 3 and equation (11)). It is visible that for none of the tested compounds, the evolution expected for $\mathrm{BA}^{49}\left(I_{\mathrm{DO}}^{-1}(T)=\right.$ const $)$ or $\mathrm{WM}^{19}\left(b=I_{\mathrm{DO}}^{-1}(T=0)=0\right)$ relations take place. Solely, patterns expected for the VFT or the 'critical-like' description occurs. Experimental results show that such patterns are associated with three 'terminal, convergence' cases: (i) $n=-(1 / b)=-1 / I_{\mathrm{DO}}^{-1}(T=0) \approx 0.17$, for ODICs and SGL (PS case); (ii) $n \approx 1.53$, for all LCs and also low-molecularweight sorbitol, propanol and polystyrene (OS case); (iii) $n \approx 1$, for DEP, EPON 828 and glycerol (NS case). Worth to mention is that the points (i) and (ii) are related to approximately the same value of the 'critical' exponent $\phi=8.5 \sim 12$ in equation (3), being remarkable that the $I_{\mathrm{DO}}^{-1}(T)$ plot allows to separate OS and PS cases.

A summary of the analysis covering 16 glass-forming systems is presented in Fig. 4 and in the Supplementary Table S1. Apart from the already-mentioned manifestation of three 'characteristic' values of the coefficient $n$, which can be related to the local symmetry, two more issues are notable. First, the clear access of the SGL system to the PS group, together with ODICs. Second, the 'intermediate' cases between three characteristic values of the coefficient $n$. For ethanol, the coefficient is $n \approx 1.2$, whereas for propanol, being a 'longer' and thus more uniaxial compound and from the same homologous series, the coefficient is $n \approx 1.5$, as for LCs and polystyrene. One of the 'intermediate' cases is cyclooctanol (plastic crystal), for which $n \approx 0.8$. In this case, the discrepancy may be linked to some distortion from the optimal positional ordering.

Consequences for the configurational entropy. Assuming the validity of equation (4), recalling equation (18) from MartinezGarcia et al..$^{52}$ and the definition of the DO index by equation (8), the following relation can be formulated:

$$
\frac{I_{\mathrm{DO}}(T)}{(1 / T)}=\frac{\partial \ln \left(E_{\mathrm{a}}(T)\right)}{\partial(1 / T)}=-\frac{1}{S_{\mathrm{C}}(T)} \frac{\partial S_{\mathrm{C}}(T)}{\partial(1 / T)}
$$



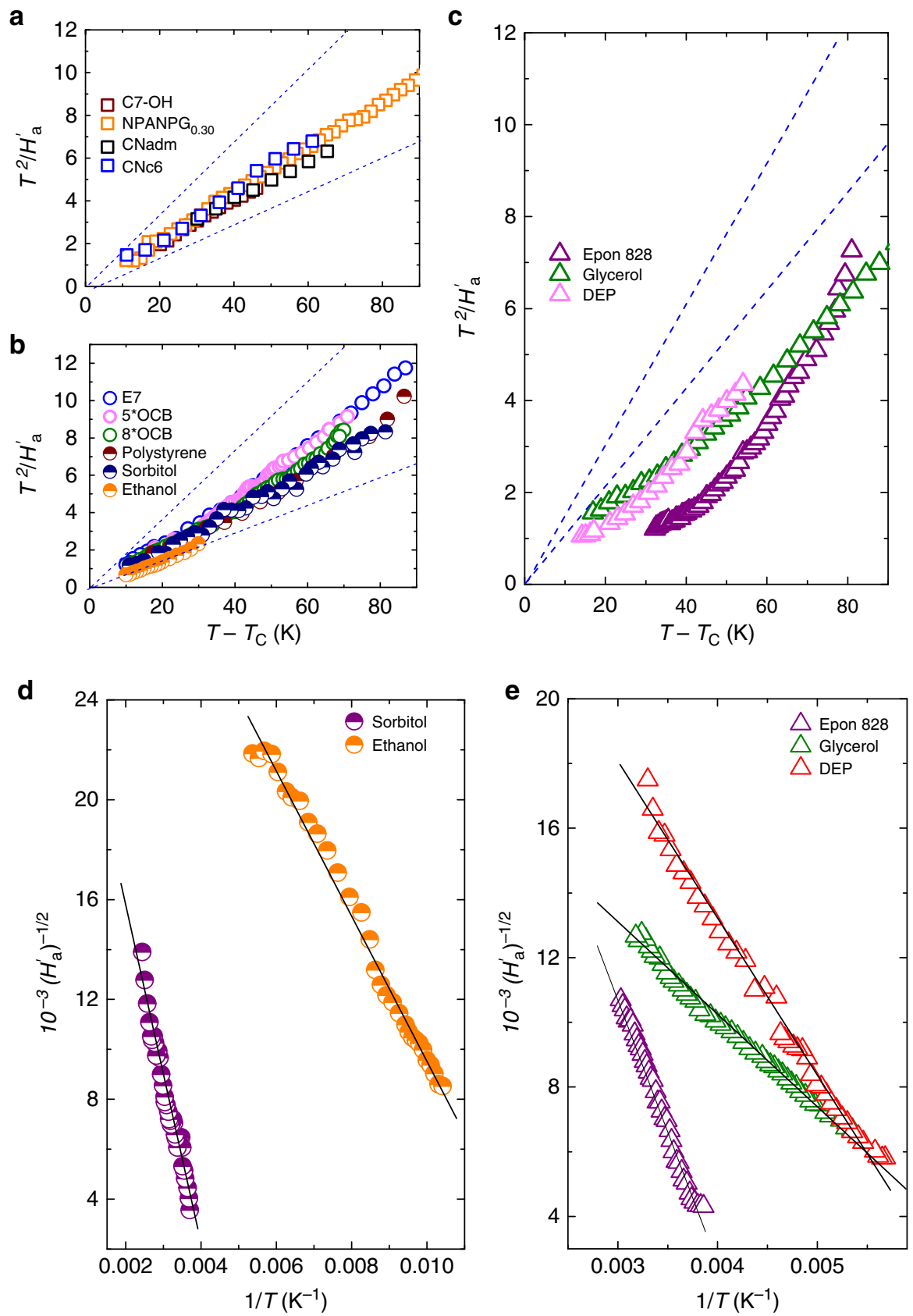

Figure 2 | Linearized derivative-based presentation of $\tau(T)$ data. Results of the linearized, derivative-based transformations ${ }^{44}$ of $\tau(T)$ experimental data, with $H_{\mathrm{a}}^{\prime}=H_{\mathrm{a}} / R$ and $H_{\mathrm{a}}=d \ln \tau / \mathrm{d}(1 / T)$. (a-c) Refer to (equations (3) and (13)). (d,e) Refer to the VFT dependence (equations (1) and (12)). The domains of the validity of the given description are shown via the linear behaviour. The blue dashed lines in a-c stand for 'terminal critical exponents' $\phi=8$ and 12, respectively.

By applying experimental findings from the previous section, the above equation can be written as:

$$
\frac{\partial S_{\mathrm{C}}(T)}{\partial(1 / T)}+\left[\frac{1}{a+b / T}\right] S_{\mathrm{C}}(T)=0
$$

The latter directly yields the following solution:

$$
S_{\mathrm{C}}(T)=S_{0}\left[1+\left(\frac{b}{a}\right)\left(\frac{1}{T}\right)\right]^{-1 / b}=S_{0}\left[1-\frac{T_{\mathrm{N}}}{T}\right]^{n}
$$

where the 'singular' temperature can be defined as $T_{\mathrm{N}}=|b / a|$ and the power exponent as $n=-1 / b=-1 /\left(I_{\mathrm{DO}}^{-1}(T=0)\right)$.

Equation (16) represents a new generalized dependence for the evolution of the configurational entropy of glass-forming liquids, allowing to construct the normalized/scaling plot shown in Fig. 5.

The scaling Fig. 5 covers the variety of ultraslowing glassforming systems ranging from low-molecular liquids and polymers to plastic crystals, LCs and SGLs. Clearly visible are three characteristic (universal?) values of the power exponent: $n \approx 1.53, n=1$ and $n \approx 0.17$, which can be linked to different dominating molecular symmetries, as shown in the right part of Fig. 5. Worth to mention is also that equation (16) has been derived by the solution of the first-order differential equation (15), without underlying assumptions that were used in the derivation of equation (5). The latter is recovered for $n=1$. It is also noteworthy that equation (16) indicates a possible link between the 'critical' temperature $\left(T_{\mathrm{C}}\right)$ in equation (3) and the VFT singular temperature $\left(T_{0}\right)$ in equation $\left.(1)\right)$. 
a

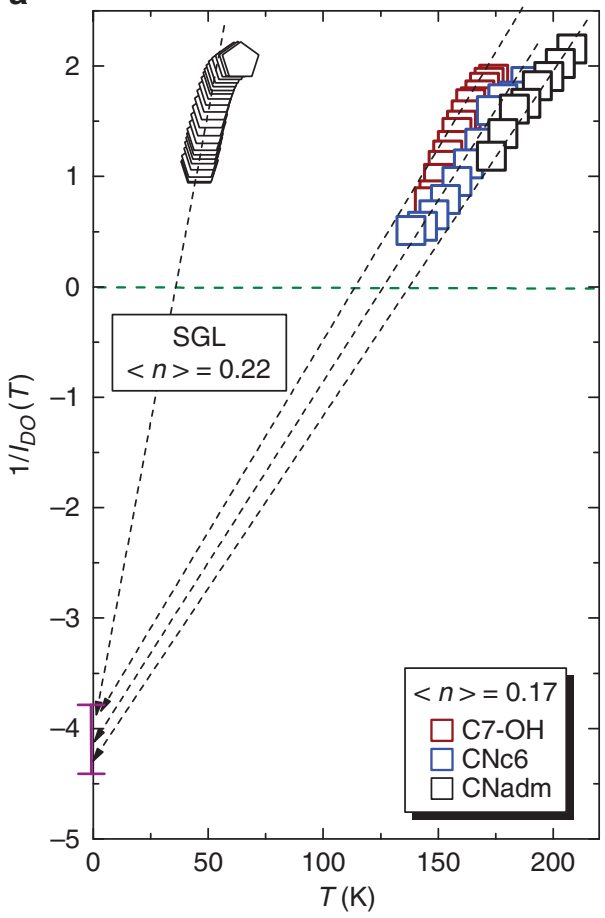

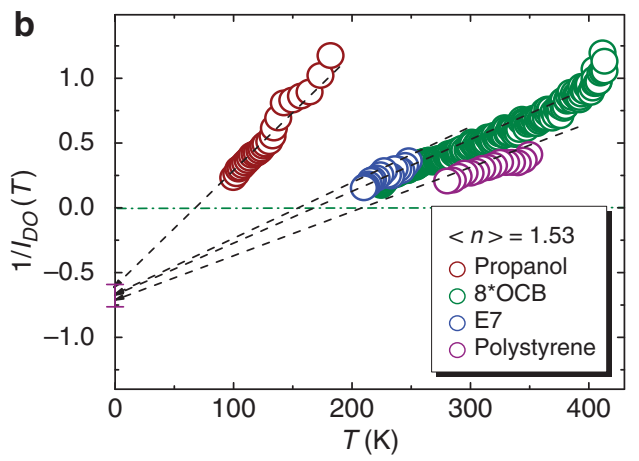

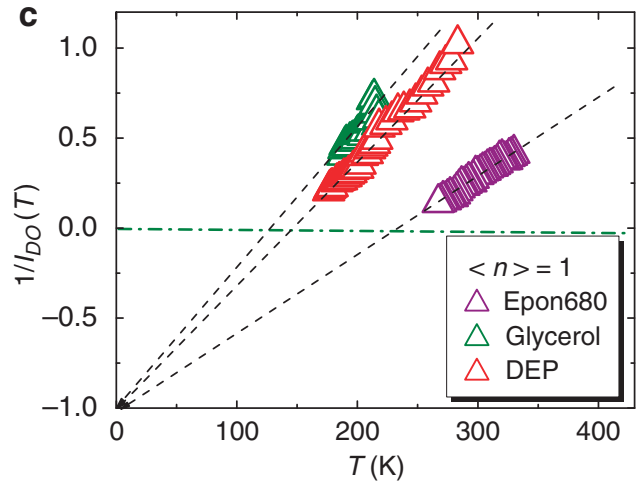

Figure 3 | Reciprocals of the experimental DO temperature index. The temperature evolution of the inverse DO index for tested glass-forming materials given in the figure. The analysis disentangles three representative cases: (a) the 'PS' (ODICs and SGL); (b) the 'OS' (LC, propanol and polystyrene); and (c) the lack of dominant symmetry (NS). Note the convergence emerging for $1 / I_{\mathrm{DO}}(T=0)$.

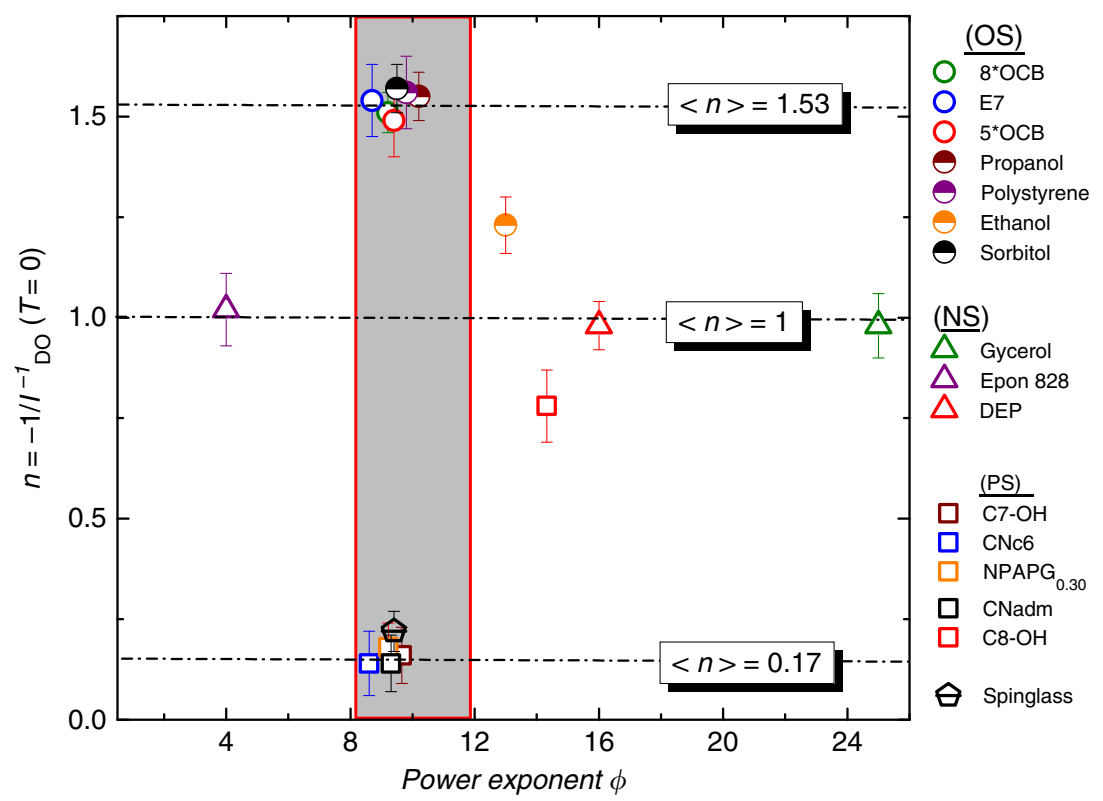

Figure 4 | Coefficient $\boldsymbol{n}$ versus the exponent $\phi$. Experimental dependencies of the symmetry coefficient ( $n$ ) versus the power exponent $\phi$ (equations (3) and (13)). For systems with the dominant VFT parameterization the exponent has been estimated as the terminal case $\phi\left(T \rightarrow T_{\mathrm{g}}\right)$. Convergence values of the coefficient $(n)$ related to VFT or 'critical-like' behaviours are indicated. The separation of OS and PS cases indicates the role of the symmetry.

\section{Discussion}

In the year 2008, Hecksher et al. ${ }^{15}$ boosted the discussion regarding one of the most fundamental experimental artifacts within glass-transition physics, namely the validity of the VFT equation, the existence of the 'final temperature divergence' $<T_{\mathrm{g}}$, and the ultimate way of $\tau(T)$ or $\eta(T)$ parameterization. This discussion and subsequent papers ${ }^{28,29,52}$ have indicated that parameterizations not associated with a 'finite temperature divergence' may be crucial. All these findings indirectly questioned the connection between the glass transition and a hidden phase transition below $T_{\mathrm{g}}$. However, parallel studies exploring the linearized derivative and distortion sensitive 


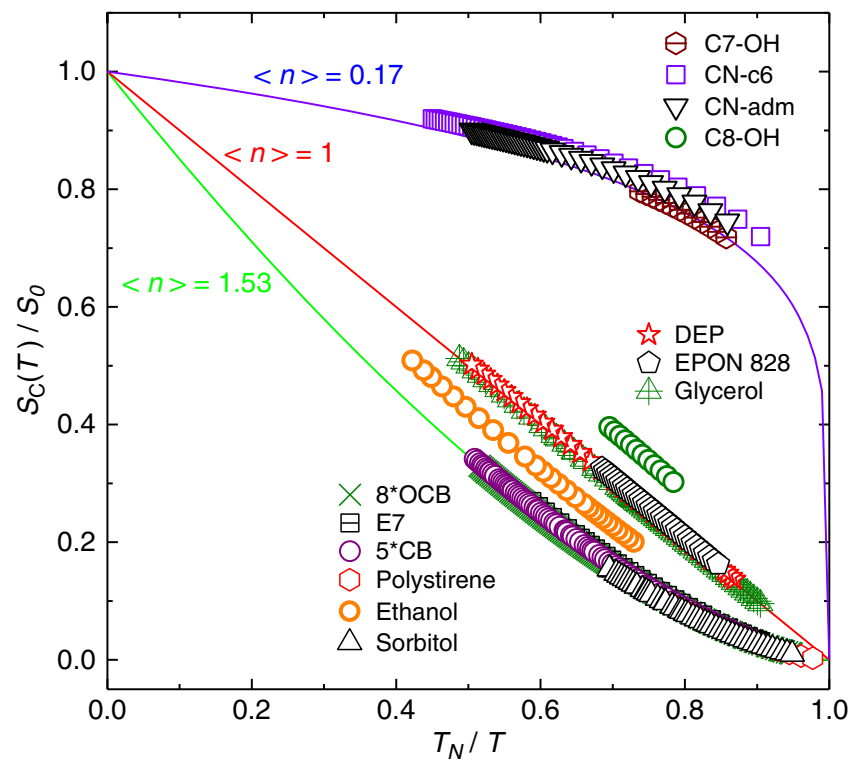

Positional Symmetry

(Critical-1)

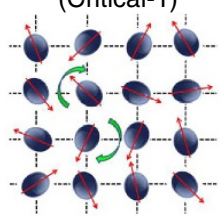

No-Symmetry(VFT)

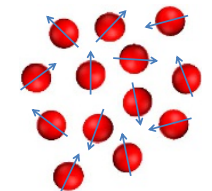

Orientational Symmetry

Critical-2

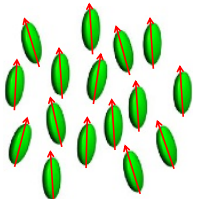

Figure 5 | Scaling configurational entropy plot. It shows the experimental evolution of the normalized structural entropy for selected experimental ultraslowing systems. The analytic evolution related to PS, NS and OS patterns (solid curves) is coupled to different 'universal' values of the coefficient exponent $n=-1 / I_{\mathrm{DO}}^{-1}(T=0)$. The related symmetries are also illustrated.

analysis led to a clear proof that the 'critical-like' description with a finite temperature divergence may be optimal for liquid crystalline glass formers, plastic crystals (not tested in Erwin and Colby ${ }^{17}$ ) and even for selected low-molecular liquids and polymers $^{27,28}$.

This paper shows that the 'finite temperature divergence' in ultraslowing glass-forming systems on approaching $T_{\mathrm{g}}$ may be feasible not only for low-molecular-weight liquids and polymers but also for LCs, plastic crystals and even SGL systems. Surprisingly, a consistency seems to be possible, despite the fact that a single equation for portraying the dynamics in the ultraslowing domain for an arbitrary glass former may not exist. It is worth stressing that the optimal parameterization via the VFT equation should be considered solely for selected compounds, and other systems such as parameterization is inherently non-optimal. This fact ought to be taken into account when validating different equations for portraying the $\tau(T)$ or $\eta(T)$ experimental behaviour.

We would like to stress the results of the analysis focused on $\mathrm{BA}^{48,49}$ and WM (MYEGA) equations ${ }^{11,19,26}$ without the finite temperature divergence.

Worth to mention is also a group of further equations of such type $e^{2,3,13,15}$, which should be tested in the future.

In conclusion, the obtained key forms for $\tau(T)$ or $\eta(T)$ parameterization can be linked to predefined values of the parameter/exponent $n=-1 / I_{\mathrm{DO}}^{-1}(T=0)$, as well as to the dominating local symmetry. They are clearly associated with a finite temperature divergence below the glass temperature. All these results indicate qualitatively new links between glass transition and critical phenomena.

\section{Methods}

Analysis of experimental data. The analysis of 'dynamic' experimental data employing higher-order derivatives of $\tau(T)$ or $\eta(T)$ experimental data is most often avoided due to scatter associated with the inherent experimental error. In this paper, this basic issue has been overcome due to the novel implementation of Savitzky-Golay (SG) filtering procedure introduced in Martinez-Garcia et al. ${ }^{52}$

Determination of the AAE from $\tau(T)$ experimental data. For estimating the DO temperature index $\left(I_{\mathrm{DO}}(T)\right)$, the experimental evolution of the AAE is needed (equation (8)). So far, it was calculated from the 'general' apparent SA equation via $E_{\mathrm{a}}(T)=R T \ln \left(\tau(T) / \tau_{0}\right)$, assuming for $\tau_{0}=10^{-14} \mathrm{~s}$, which is most often considered as the 'typical' or 'universal' value, at least for ultraviscous liquids. Notwithstanding, there is clear evidence that the value of $\tau_{0}$ ranges from $10^{-11}-10^{-16} \mathrm{~s}$ (ref. 49). The erroneous value $\tau_{0}$ for a given experimental system can notably influence estimations of $E_{\mathrm{a}}(T)$. In this paper, experimental values of $E_{\mathrm{a}}(T)$ were calculated in a novel way, avoiding $\tau_{0}$-related problems. The AAE was calculated via $H_{\mathrm{a}}^{\prime}(T)=H_{\mathrm{a}}(T) / R=\mathrm{d} \ln \tau / d(1 / T)$. Ultimate estimation was supported by the SG filtering procedure. Subsequently, the following 'general' first-order differential equation was solved:

$$
\begin{gathered}
\frac{\partial E_{a}^{\prime}(1 / T)}{\partial(1 / T)}+\frac{E_{a}^{\prime}(1 / T)}{(1 / T)}=\frac{H_{a}^{\prime}(1 / T)}{(1 / T)} \\
\left\{(1 / T) \rightarrow \infty: E_{a}(0), E_{a}\left(T_{N}\right) \rightarrow \infty\right\} \\
\left\{(1 / T) \rightarrow 0: E_{a}=c t e\right\}
\end{gathered}
$$

The 'noise-related' scatter of the calculated $E_{\mathrm{a}}(T)$ values was minimized by the SG filtering procedure for each tested glass-forming system. In a next step, the DO was calculated via the equation (8).

Derivations of the DO temperature indexes for selected equations describing $\tau(T)$ or $\eta(T)$ evolutions. The form of $I_{\mathrm{DO}}(T)$ for VFT equation (1) and BA relation $\tau(T)=\tau_{0} \exp \left(A / T^{\mathrm{D}}\right)$ can be found in Erwin and Colby ${ }^{17}$. The DO temperature index for the WM (MYEGA) equation can be obtained by linking equation (2), the SA equation and $I_{\mathrm{DO}}(T)$ definition (equation (8)):

$$
E_{\mathrm{a}}(T)=R K \exp \left(\frac{C}{T}\right) \text {, and then } I_{\mathrm{DO}}^{\mathrm{WM}}=-\frac{T}{E_{\mathrm{a}}(T)} \frac{\partial E_{\mathrm{a}}(T)}{\partial T}=-T \partial(C / T)=\frac{C}{T}
$$

Forms of $I_{\mathrm{DO}}(T)$ for the critical-like case in the ultraslowing/ultraviscous dynamic domain can be obtained by linking equation (3) and the general SA dependence $\tau(T)=\tau_{0} \exp \left(E_{\mathrm{a}}^{\prime}(T) / R T\right):$

$$
E_{\mathrm{a}}^{\prime}(T)=R T\left[-\phi \ln \left(\frac{T-T_{\mathrm{C}}}{T_{\mathrm{C}}}\right)+\ln \left(\frac{\tau_{\mathrm{C}}}{\tau_{0}}\right)\right]=R T\left[\ln \left(\frac{T T_{\mathrm{C}}}{\left(T-T_{\mathrm{C}}\right) T}\right)^{\phi}+\ln \frac{\tau_{\mathrm{C}}}{\tau_{0}}\right]
$$

$$
E_{\mathrm{a}}(T)=E_{\mathrm{a}}^{\prime}(T) / R=T \ln \left(\frac{T}{T-T_{\mathrm{C}}}\right)^{\phi}+T \ln \left(\frac{T_{\mathrm{C}}}{T}\right)^{\phi}+C T
$$

This work focuses on the ultraviscous/ultraslowing dynamic domain close to $T_{\mathrm{g}}$, that is, for $T \rightarrow T_{\mathrm{C}}$, hence:

$$
E_{\mathrm{a}}(T) \approx T \ln \left(\frac{T}{T-T_{\mathrm{C}}}\right)^{\phi}
$$



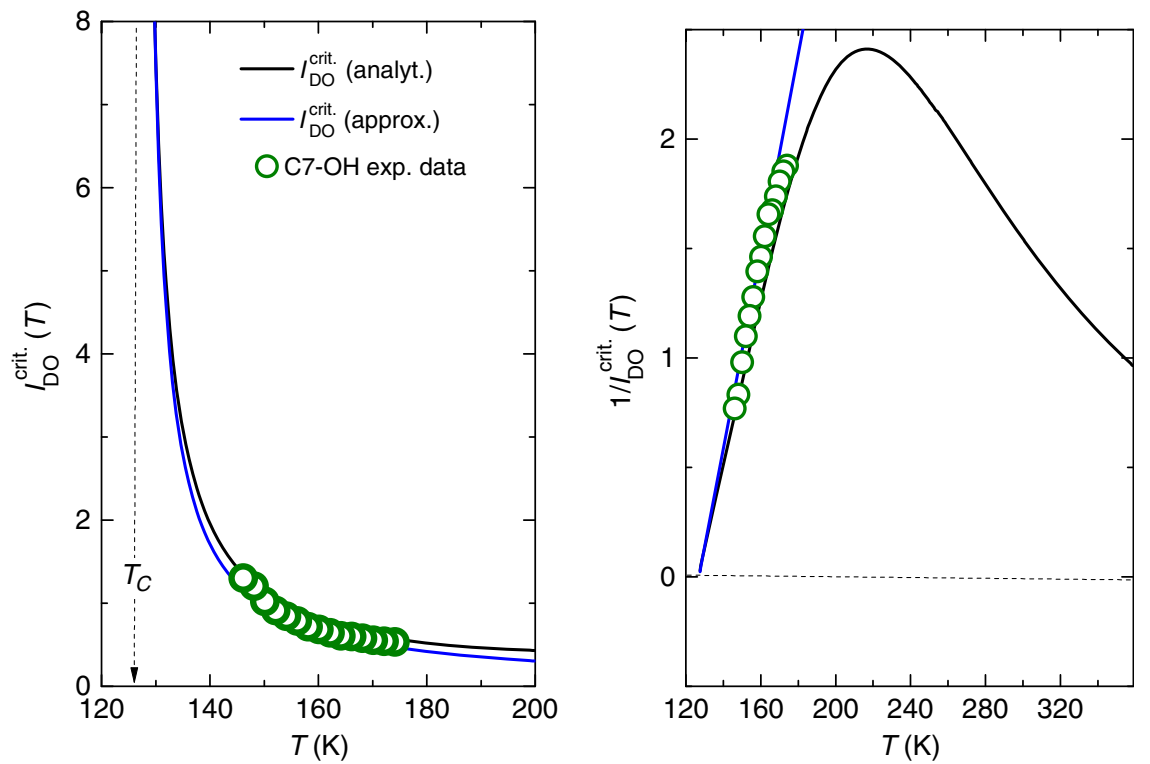

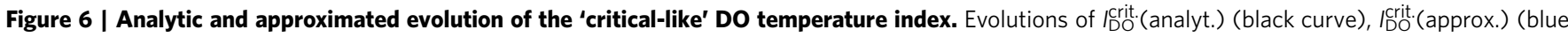
curve) and their reciprocals (right side), compared with the empirical DO index for C7-OH (ODIC, plastic crystal) where the critical-like $\tau(T)$ evolution is optimal.

The latter equation can be re-written as:

$$
E_{\mathrm{a}}(T)=T T_{\mathrm{C}} \ln \left(\frac{T}{T-T_{\mathrm{C}}}\right)^{\phi / T_{\mathrm{C}}}=T T_{\mathrm{C}} \delta(T)
$$

The function $\delta(T)=\ln \left[T /\left(T-T_{\mathrm{C}}\right)\right]^{\phi / T_{\mathrm{C}}}$ can be expanded into the Taylor series because of its inherent features $\left(\phi / T_{\mathrm{C}} \ll 1\right)$ if $|\delta(T)-1| \leq 1$. This condition is valid for ultraslowing ODICs, LCs and SGL systems tested in this paper. Taking this into account, one obtains:

$$
E_{\mathrm{a}}(T)=T T_{\mathrm{C}} \sum_{i=1}^{\infty} \frac{(-1)^{i+1}}{i}\left[\left(\frac{T}{T-T_{\mathrm{C}}}\right)^{\phi / T_{\mathrm{C}}}-1\right]^{i} \approx T T_{\mathrm{C}}\left(\frac{T}{T-T_{\mathrm{C}}}\right)^{\phi / T_{\mathrm{C}}}
$$

Then, recalling $I_{\mathrm{DO}}(T)$ definition (equation (8)):

$$
\left.I_{\mathrm{DO}}(T)=I_{\mathrm{DO}}^{\text {crit. }} T\right)=\left(\frac{\left(\frac{T}{T-T_{\mathrm{c}}}\right)}{\frac{1}{\varphi} \ln \left(\frac{\tau_{\mathrm{c}}}{\tau_{\mathrm{o}}}\right)-\ln \left[\frac{T-T_{\mathrm{c}}}{T_{\mathrm{c}}}\right]}-1\right) \approx \frac{\phi}{T-T_{\mathrm{c}}}
$$

The evolutions of $I_{\mathrm{DO}}^{\text {crit. }}(T)$ and $1 / I_{\mathrm{DO}}^{\text {crit. }}(T)$ are presented graphically in Fig. 6. The analytic form of the index is given in the bracket in equation (24) and shown graphically in Figure 6, together with the approximation $I_{\mathrm{DO}}^{\text {crit. }}=I_{\mathrm{DO}}^{\text {crit. }}($ approx. $)=\phi /\left(T-T_{\mathrm{C}}\right)$. This figure is supplemented by the experimental DO index for C7-OH (ODIC; plastic crystal) where the critical-like parameterization of $\tau(T)$ is optimal (see Fig. 2). The validity of $I_{\mathrm{DO}}^{\text {crit. }}$ (approx.) for portraying experimental data in the ultraslowing domain (below $T_{\mathrm{B}}$ ) is clearly visible.

\section{References}

1. Kennedy, D. 125th Anniversary Issue: 125 outstanding problems in all of science: what is the nature of the glassy state. Science 309, 83 (2005).

2. Jones, R. A. L. Introduction to Soft Matter (Oxford University Press, NY, 2005).

3. Donth, E. The Glass Transition. Relaxation Dynamics in Liquids and Disordered Materials. Vol. 48 (Springer Series in Material Sci. II, Springer, Berlin, 1998).

4. Poirier, J.-P. Introduction to the Physics of the Earth's Interior (Cambridge University, Cambridge, 2000).

5. Mezzenga, R., Schurtenberger, P., Burbidge, A. \& Michel, M. Understanding foods as soft materials. Nat. Mater. 4, 729-740 (2005).

6. Andersen, H. C. Molecular dynamics studies of heterogeneous dynamics and dynamic crossover in supercooled atomic liquids. Proc. Natl Acad. Sci. USA 102, 6686-6691 (2005).

7. Lubchenko, V. \& Wolyness, P. G. Theory of Structural Glasses and Supercooled Liquids. Ann. Rev. Phys. Chem. 58, 235-266 (2007).

8. Kivelson, S. A. \& Tarjus, G. In search of a theory of supercooled liquids. Nat. Mater. 7, 831-833 (2008).

9. Roland, C. M. Characteristic relaxation times and their invariance to thermodynamic conditions. Soft Matter 4, 2316-2322 (2008).
10. Hedges, L. O., Jack, R. L., Garrahan, J. \& Chandler, D. A. Dynamic orderdisorder in atomistic models of structural glass formers. Science 6, 1309-1313 (2009).

11. Mauro, J. C. Through a glass, darkly: dispelling three common misconceptions in glass science. Int. J. Appl. Glass Sci. 2, 245-261 (2011).

12. Angell, A. \& Klein, I. S. Glass physics: prigogine and defay say relax. Nat. Phys. 7, 750-751 (2011).

13. Ngai, K. L. Relaxation and Diffusion in Complex Systems (Springer, Berlin, 2011).

14. Tanaka, H. Bond orientational order in liquids: Towards a unified description of water-like anomalies, liquid-liquid transition, glass transition, and crystallization. Europ. Phys. J. E 35 colloquium 1-84 (2012).

15. Hecksher, T., Nielsen, A. I., Olsen, N. B. \& Dyre, J. C. Little evidence for dynamic divergences in ultraviscous molecular liquids. Nat. Phys. 4, 737-741 (2008).

16. Colby, R. H. Dynamic scaling approach to glass formation. Phys. Rev. E 61, 1783-1792 (2000).

17. Erwin, B. M. \& Colby, R. H. Temperature dependence of relaxation times and the length scale of cooperative motion for glass-forming liquids. J. Non-Cryst. Solids 307, 225-231 (2002).

18. Tanaka, H., Kawasaki, T., Shintani, H. \& Watanabe, K. Critical-like behaviour of glass-forming liquids. Nat. Mater. 9, 324-331 (2010).

19. Mauro, J. C., Yue, Y., Ellison, A. J., Gupta, P. K. \& Allan, D. C. Viscosity of glass-forming liquids. Proc. Natl Acad. Sci. USA 106, 19780-19784 (2009).

20. Lunkenheimer, P., Kastner, S., Köhler, M. \& Loidl, A. Temperature development of glassy $\alpha$-relaxation dynamics determined by broadband dielectric spectroscopy. Phys. Rev. E 81, 051504 (2010).

21. Martinez-Garcia, J. C., Tamarit, J. Ll. \& Rzoska, S. J. Enthalpy space analysis of the evolution of the primary relaxation time in ultraslowing systems. J. Chem. Phys. 134, 024512-024517 (2011).

22. Vogel, H. Temperaturabhängigkeitsgesetz der Viskosität von Flüssigkeiten. Phys. Zeit 22, 645-646 (1921).

23. Fulcher, G. S. Analysis of recent measurements of the viscosity of glasses. J. Am. Ceram. Soc. 8, 339-355 (1925).

24. Tammann, G. Glasses as supercooled liquids. J. Soc. Glass Technol. 9, 166-185 (1925).

25. McKenna, G. B. Diverging views on glass transition. Nat. Phys. 4, 673-674 (2008).

26. Waterton, S. C. The viscosity-temperature relationship and some inferences on the nature of molten and of plastic glass. J. Soc. Glass Technol. 16, 244-247 (1932).

27. Rouxel, T. Thermodynamics of viscous flow and elasticity of glass forming liquids in the glass transition range. J. Chem. Phys. 135, 184501-184516 (2011).

28. Drozd-Rzoska, A., Rzoska, S. J. \& Paluch, M. Universal critical-like scaling of dynamic properties in symmetry-selected glass formers. J. Chem. Phys. 129, 184509-184515 (2008). 
29. Drozd-Rzoska, A., Rzoska, S. J., Pawlus, S., Martinez-Garcia, J. C. \& Tamarit, J. -L. Evidence for critical-like behavior in ultraslowing glass-forming systems. Phys. Rev. E 82, 031501 (2010).

30. Adam, G. \& Gibbs, J. H. On the temperature dependence of cooperative relaxation properties in glass-forming liquids. J. Chem. Phys. 43, 139-146 (1965).

31. Angell, C. A. Entropy and fragility in supercooling liquids. J. Res. Nat. Inst. Stand. Technol. 102, 171-185 (1997).

32. Richert, R. \& Angell, C. A. Dynamics of glass-forming liquids. V. On the link between molecular dynamics and configurational entropy. J. Chem. Phys. 108, 9016-9027 (1998)

33. Martinez, L.-M. \& Angell, C. A. A thermodynamic connection to the fragility of glass-forming liquids. Nature 410, 663-667 (2001)

34. Kauzmann, W. The nature of the glassy state and the behavior of liquids at low temperatures. Chem. Rev. 43, 219-256 (1948).

35. Tanaka, H. Relation between thermodynamics and kinetics of glass-forming liquids. Phys. Rev. Lett. 90, 05570 (2003).

36. Eckmann, J. P. \& Procaccia, I. Ergodicity and slowing down in glass-forming systems with soft potentials: no finite-temperature singularities. Phys. Rev. E 78, 011503 (2008).

37. Richert, R. Scaling vs. Vogel-Fulcher-type structural relaxation in deeply supercooled materials. Physica A 287, 26-36 (2000).

38. Stanley, H. E. Introduction to Phase Transitions and Critical Phenomena (Oxford Univ. Press., NY, 1987).

39. Anisimov, M. A. Critical Phenomena in Liquids and in Liquid Crystals (Gordon and Breach, Reading, 1992).

40. Angell, C. A. in Relaxations in Complex Systems (ed. Ngai, K. L.) 3NRL, (Washington, DC, 1985).

41. Böhmer, R., Ngai, K. L., Angell, C. A. \& Plazek, J. D. Nonexponential relaxations in strong and fragile glass formers. J. Chem. Phys. 99, 4201-4209 (1993).

42. Dyre, J. C. \& Olsen, N. B. Landscape equivalent of the shoving model. Phys. Rev. E 69, 042501 (2004).

43. Grüneisen, E. Theorie des festen Zustandes einatomiger elemente. Ann. Phys. 12, 257-306 (1912)

44. Drozd-Rzoska, A. \& Rzoska, S. J. On the derivative-based analysis for temperature and pressure evolution of dielectric relaxation times in vitrifying liquids. Phys. Rev. E 73, 041502 (2006).

45. Wang, L.-M. \& Mauro, J. C. An upper limit to kinetic fragility in glass-forming liquids. J. Chem. Phys. 134, 044522 (2011).

46. Simon, S. L., Plazek, D. J., Sobieski, J. W. \& McGregor, E. T. Physical aging of a polyetherimide: Volume recovery and its comparison to creep and enthalpy measurements. J. Polym. Sci. Part B. Polym. Phys. 35, 929-936 (1997).

47. McKenna, G. B. Deformation and flow of matter: Interrogating the physics of materials using rheological methods. J. Rheol. 56, 113-158 (2012).

48. Bässler, H. Viscous flow in supercooled liquids analysed in terms of transport theory for random media with energetic disorder. Phys. Rev. Lett. 58, 767-770 (1987).

49. Avramov, I. Viscosity in disordered media. J. Non-Cryst. Solids 351, 3163-3173 (2005).

50. Stickel, F., Fischer, E. W. \& Richert, R. Dynamics of glass-forming liquids. I. Temperature-derivative analysis of dielectric relaxation data. J. Chem. Phys. 102, 6251 (1995).
51. Richert, R. \& Angell, C. A. Dynamics of glass-forming liquids. V. On the link between molecular dynamics and configurational entropy. J. Chem. Phys. 108, 9016-9026 (1998).

52. Martinez-Garcia, J. C., Martinez-Garcia, J., Rzoska, S. J. \& Hulliger, J. The new insight into dynamic crossover in glass forming liquids from the apparent enthalpy analysis. J. Chem. Phys. 137, 064501 (2012).

53. Martinez-Garcia, J. C. et al. Disentangling the secondary relaxations in the orientationally disordered mixed crystals: cycloheptanol + cyclooctanol twocomponent system. J. Phys. Chem. B 114, 6099-6106 (2010).

54. Brand, R., Lunkenheimer, P., Schneider, U. \& Loidl, A. Excess wing in the dielectric loss of glass-forming ethanol: a relaxation process. Phys. Rev. B 62, 8878-8883 (2000).

55. Schwartz, A., Colmenero, J. \& Alegria, A. Dielectric study of the segmental relaxation of low and high molecular weight polystyrenes under hydrostatic pressure. J. Non-Cryst. Solids 353, 4298-4302 (2007).

56. Drozd-Rzoska, A., Rzoska, S. J., Pawlus, S. \& Tamarit, J.Ll. Dynamics crossover and dynamic scaling description in vitrification of orientationally disordered crystal. Phys. Rev. B 73, 224205 (2006).

57. Jönsson, P., García-Palacios, J. L., Hansen, M. F. \& Norblad, P. Relaxation in interacting nanoparticle systems. J. Mol. Liq. 114, 131-140 (2004).

58. Martinez-Garcia, J. C. et al. Universal critical-like scaling of dynamics plastic crystals. J. Non-Cryst. Solids 357, 329-333 (2011).

59. Martinez-Garcia, J. C. et al. $\alpha$-relaxation dynamics of orientanionally disordered mixed crystals composed of $\mathrm{Cl}$-adamantane and $\mathrm{CN}$-adamantane. J. Chem. Phys 132, $164516(2010)$

\section{Acknowledgements}

S.J.R. and A.D.-R. were supported by the Grant no. N N202 231737 from National Centre for Science (NCN, Poland). We would like to thank Iris Martinez-Heer for her valuable contribution to improve the final version of the article.

\section{Author contributions}

J.C.M.-G. and S.J.R. conceived the method and created the final shape of the paper. A.D.R. and S.J.R. carried out experiments associated with some sets of experimental data A.D.R. yielded the linearized derivative-based analysis. J.M.-G. was responsible for the Savitzky-Golay methodology.

\section{Additional information}

Supplementary Information accompanies this paper at http://www.nature.com/ naturecommunications

Competing financial interests: The authors declare no competing financial interests.

Reprints and permission information is available online at http://npg.nature.com/ reprintsandpermissions/

How to cite this article: Martinez-Garcia, J. C. et al. A universal description of ultraslow glass dynamics. Nat. Commun. 4:1823 doi: 10.1038/ncomms2797 (2013).

This work is licensed under a Creative Commons AttributionNonCommercial-ShareAlike 3.0 Unported License. To view a copy of this license, visit http://creativecommons.org/licenses/by-nc-sa/3.0/ 